\title{
La digitalización de la experiencia enoturística: una revisión de la literatura y aplicaciones prácticas
}

\section{Digitalization of wine Tourism Experience: a literature review and practical applications}

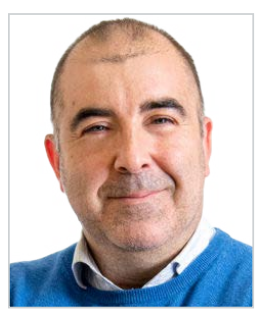

Gorka Zamarreño Aramendia. Doctor en Ciencias de la Comunicación y profesor Asociado de Historia Económica en la Universidad de Málaga, desarrolla una de sus líneas de investigación del Turismo en el Franquismo a través del cine, así como la capacidad que tiene las producciones cinematográficas como herramienta para la docencia y como generador de un relato que es capaz de transformar la percepción del hecho histórico. También se han publicado diversos artículos sobre la importancia histórica de la marca territorio y la relevancia del sector vitivinícola en la provincia de Málaga. Es miembro del grupo de Investigación Mediterráneo Económico con líneas de estudio relacionadas con la diversidad turística a lo largo del siglo XX y relativas al siglo XXI en el turismo de fronteras y el turismo del vino.

Universidad de Málaga, España

gzama@uma.es

ORCID: 0000-0003-1568-4540

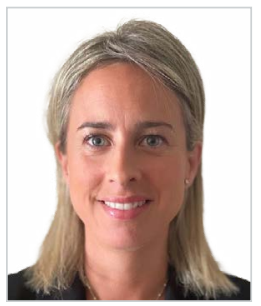

Elena Cruz Ruíz. Doctora en Economía, Licenciado en Administración de Empresas por la Universidad de Málaga (España), Máster en Turismo. Profesora del Departamento de Ciencias Económicas y Empresariales de la Facultad de Ciencias Económicas y Empresariales de la Universidad de Málaga. Miembro del Grupo Andaluz de Investigación y Desarrollo Tecnológico 'Mediterráneo Económico' (SEJ-121) y del Comité Científico del Foro por la Paz del Mediterráneo. Líneas de investigación: las distintas modalidades del sector turístico: Cruceros, Gastronomía, Enología, etc. Experto en temas relacionados con la marca territorio. Actualmente, dirige dos proyectos de investigación a través de la Oficina de Transferencia de la Universidad de Málaga (OTRI) con el fin de analizar la percepción de turistas y residentes de la marca Málaga.

Universidad de Málaga, España

ecruz@uma.es

ORCID: 0000-0002-8334-3900

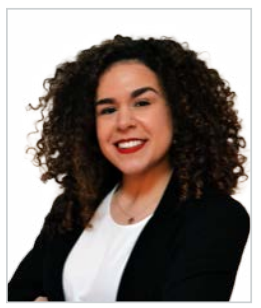

Claudia Hernando Nieto. Licenciada en Economía y Administración de Empresas, investigadora predoctoral en área de experiencia de cliente trabaja en la actualidad en el Departamento de Marketing y Comunicación de GSK.

Universidad de Málaga, España

claunandez@uma.es

ORCID: 0000-0003-2709-1638

Cómo citar este artículo:

Zamarreño Aramendia, G.; Cruz Ruíz, E. y Hernando Nieto, C. (2021). La digitalización de la experiencia enoturística: una revisión de la literatura y aplicaciones prácticas. Doxa Comunicación, 33, pp. 257- 283.

https://doi.org/10.31921/doxacom.n33a930 
Recibido: 18/01/2021 - Aceptado: 16/05/2021 - En edición: 03/09/2021 - Publicado: 01/12/2021

\section{Resumen:}

La evolución tecnológica unida al uso de las tecnologías TICs y otros recursos de comunicación digitales como la realidad virtual, aumentada y la tecnología 4D están transformando el ciclo de la experiencia turística. El turismo del vino debe adaptarse para que una experiencia enoturística sea inolvidable y por lo tanto sea recomendada debe ser innovadora y multisensorial. Se ha realizado un análisis bibliométrico en base a la literatura científica en relación con la digitalización de la experiencia enoturística y el uso de los recursos de comunicación digital. Se examinaron un total de 72 documentos académicos que arrojan como resultado que los productores pueden incrementar el valor de la experiencia hedónica al acercar la bodega al cliente antes de realizar la visita, durante la misma y en la post-visita. Si la experiencia de todo el proceso es satisfactoria, se consigue un E-WOM (boca a boca digital) positivo y la creación de una comunidad de marca leal.

\section{Palabras clave:}

Enoturismo y realidad virtual; digitalización y TiCs; ciclo de la experiencia turística; marketing del vino.
Received: 18/01/2021 - Accepted: 16/05/2021 - Early access: 03/09/2021 - Published: 01/12/2021

\section{Abstract:}

Technological evolution coupled with the use of ICT technologies and other digital communication resources, such as virtual reality, augmented reality and $4 D$ technology, is transforming the tourism experience cycle. Wine tourism must adapt in order for a wine tourism experience to be unforgettable and, therefore, recommended, so it must be innovative and multisensory. A bibliometric analysis has been carried out based on the scientific literature related to the digitalization of the wine tourism experience and the use of digital communication resources. A total of 72 academic papers were examined, with the result that producers can increase the value of the hedonic experience by bringing the winery closer to the customer before, during and after the visit. A successful experience throughout the whole process leads to positive E-WOM (digital word of mouth) and to the creation of a loyal brand community.

\section{Keywords:}

Wine tourism and virtual reality; digitalization and ITCs; tourism experience cycle; wine marketing.

\section{Introducción}

En los últimos años, el uso de internet y las tecnologías de la información y la comunicación (TICs) han dado lugar a la configuración de una nueva economía, dónde la globalización ha dejado paso a la digitalización que ha revolucionado la forma en que se crean y consumen las experiencias. El sector turístico debido a su carácter internacional ha asistido al nacimiento de nuevos modelos de negocio, mercados y técnicas de comunicación y venta, (Buhalis, 1993, 1998; Buhalis, Jafari, y Werthner, 1997; Law, Buhalis, y Cobanoglu, 2014; Lewis y Bridger, 2001) cambiando totalmente la forma de interactuar del cliente con el destino (Mak, 2017).

Las tecnologías ofrecen la posibilidad de que los turistas accedan a la información y establecen un nexo de contacto directo con los proveedores de servicios turísticos, además de generar experiencias más ricas, participativas e inmersivas, teniendo como resultado un aumento de la satisfacción de los usuarios.

El enoturismo es definido como la visita a bodegas, viñedos, festivales o ferias con la principal motivación de catar la uva y su producto en la región donde se produce (Hall et al., 2000). Getz (2000) introduce el concepto de nicho de mercado, de modo que combina una forma de comportamiento del consumidor con una estrategia de destino, lo que supone una oportunidad para la creación de imagen de marca y de marketing para las empresas vinícolas. 
El objetivo del estudio es dar a conocer al lector las aportaciones académicas más importantes, realizadas en torno a la digitalización de la experiencia enoturística, mencionando algunas experiencias prácticas relevantes, que han sido puestas en marcha por distintas bodegas. Para ello se ha examinado el marco general sobre el turismo del vino, analizando la literatura sobre el enoturismo y la utilización de las TIC en las bodegas. En un segundo epígrafe, se han abordado las cuestiones metodológicas para analizar el impacto de las tecnologías en el proceso de la experiencia del turista. Los resultados muestran la adaptación de las bodegas a este fenómeno, convirtiéndose en sitios de producción y en focos de atracción turística. Finalmente, en el apartado de conclusiones se presentan una serie de reflexiones sobre la digitalización del turismo del vino.

\section{Metodología}

Las revisiones de la literatura son de gran utilidad en multitud de campos de investigación, destacando las ciencias sociales, de modo que, sirven para arrojar luz sobre los entresijos científicos (Mulrow, 1994). La investigación documental puede ser ordenada mediante una revisión de la literatura, pero se deben señalar ciertas limitaciones a esta metodología según algunos especialistas como Briner y Walshe (2014). Boote y Beile (2005) inciden en que la revisión de la literatura puede considerarse como el primer paso para realizar investigaciones significativas. El objetivo y el alance de la misma, toma como base los planteamientos sugeridos por Hart (1998) y Montella (2017). El primer paso de la revisión fue la identificación de los propósitos de la búsqueda. La principal pregunta de investigación que se aborda es: ¿cuál es el estado del arte sobre la digitalización de la experiencia enoturística? El segundo paso fue identificar las palabras clave y fuentes. La búsqueda se centró en la utilización de las páginas web, las redes sociales, la realidad virtual y aumentada y el uso de los códigos QR, como aspectos relevantes en la experiencia de digitalización de las visitas enoturísticas.

Al identificar las palabras clave, también se tuvieron en cuenta los sinónimos y posteriormente se ha realizado un análisis. La Tabla 1 muestra la combinación de palabras clave elegidas hasta el año 2020.

Tabla 1. Palabras clave en las bases de datos de Web of Science (WOS) y Scopus

\begin{tabular}{|c|c|c|c|c|c|}
\hline & Palabras clave & Dónde & Base de datos & $\begin{array}{c}\text { Tipo de } \\
\text { documento }\end{array}$ & Resultados \\
\hline 1 & Wine and social media & Tem, Titl,Ab,Keyw & WOS & Todos & 129 \\
\hline 2 & Wine and website & Tem, Titl,Ab,Keyw & WOS & Todos & 249 \\
\hline 3 & Wine and virtual reality & Tem, Titl,Ab,Keyw & WOS & Todos & 60 \\
\hline 4 & Wine and augmented reality & Tem, Titl,Ab,Keyw & WOS & Todos & 5 \\
\hline 5 & Wine and QR Code & Tem, Titl,Ab,Keyw & WOS & Todos & 3 \\
\hline 6 & Wine tourism and social media & Tem, Titl,Ab,Keyw & WOS & Todos & 12 \\
\hline 7 & Wine tourism and website & Tem, Titl,Ab,Keyw & WOS & Todos & 20 \\
\hline
\end{tabular}




\begin{tabular}{|c|c|c|c|c|c|}
\hline 8 & $\begin{array}{l}\text { Wine tourism and virtual } \\
\text { reality }\end{array}$ & Tem, Titl,Ab,Keyw & WOS & Todos & 1 \\
\hline 9 & $\begin{array}{l}\text { Wine tourims and augmented } \\
\text { reality }\end{array}$ & Tem, Titl,Ab,Keyw & wOS & Todos & 0 \\
\hline 10 & Wine tourism and QR Code & Tem, Titl,Ab,Keyw & WOS & Todos & 1 \\
\hline 11 & Wine and social media & Ab,Titl, Keyw & Scopus & Todos & 107 \\
\hline 12 & Wine and website & Ab,Titl, Keyw & Scopus & Todos & 161 \\
\hline 13 & Wine and virtual reality & Ab,Titl, Keyw & Scopus & Todos & 41 \\
\hline 14 & Wine and augmented reality & Ab,Titl, Keyw & Scopus & Todos & 0 \\
\hline 15 & Wine and QR Code & Ab,Titl, Keyw & Scopus & Todos & 5 \\
\hline 16 & Wine tourism and social media & Ab,Titl, Keyw & Scopus & Todos & 20 \\
\hline 17 & Wine tourism and website & Ab,Titl, Keyw & Scopus & Todos & 19 \\
\hline 18 & $\begin{array}{l}\text { Wine tourism and virtual } \\
\text { reality }\end{array}$ & Ab,Titl, Keyw & Scopus & Todos & 1 \\
\hline 19 & $\begin{array}{l}\text { Wine tourims and augmented } \\
\text { reality }\end{array}$ & Ab,Titl, Keyw & Scopus & Todos & 1 \\
\hline 20 & Wine tourism and QR Code & Ab,Titl, Keyw & Scopus & Todos & 1 \\
\hline
\end{tabular}

Fuente: elaboración propia. Tem (Tema), Titl (Título), Ab (Abstract), Keyw (Palabras Clave)

Para recoger todo el espectro de posibles artículos académicos de relevancia se recurrió a las dos bases de datos más importantes, la Web of Science (WOS) y Scopus que se completaron con otras fuentes académicas que se centran en el mundo del vino, como el Journal of Wine Business Research, el Journal of Wine Research, el Journal of Wine Economics and Policy, AWBR y la AAWE. La combinación de múltiples fuentes, como sugiere Tranfield et al. (2003) ha ampliado el número de fuentes examinadas.

Se han examinado un total de 72 documentos académicos y un $82 \%$ del total fueron publicados a partir de 2010. En la tabla 2 se muestran los principales resultados agrupados por los diferentes conceptos que engloban las TiCs. Cabe destacar que la selección se realizó con el fin de presentar trabajos que estén específicamente dirigidos al negocio del enoturismo. Otra aportación que nos permite esta revisión de la literatura es en los que se analiza el impacto de la realidad virtual, aumentada y su combinación en la experiencia del consumidor (Flavián et al., 2018) y el desarrollo de modelos de experiencia virtual multisensorial para el turismo del vino. 
Finalmente, se identifica el concepto de turismo inteligente en la gestión del enoturismo, que engloba las infraestructuras que permiten la administración del turismo a través de los recursos digitales de comunicación, de modo que fomenten oportunidades inteligentes e incrementen la eficiencia de los negocios turísticos y el valor que el cliente da a la experiencia. (Gretzel, Zhong y Koo, 2016).

Tabla 2. Estudios analizados y clasificados por conceptos

\begin{tabular}{|c|c|c|c|c|}
\hline Referencias & $\begin{array}{l}\text { Página } \\
\text { Web }\end{array}$ & $\begin{array}{l}\text { Redes } \\
\text { Sociales }\end{array}$ & $\begin{array}{l}\text { Realidad } \\
\text { Virtual y } \\
\text { Realidad } \\
\text { Aumentada }\end{array}$ & $\begin{array}{l}\text { Códigos } \\
\text { QR }\end{array}$ \\
\hline $\begin{array}{l}\text { Abd-Elaziz, M. E., Aziz, W. M., Khalifa, G. S., Abdel-Aleem, M. (2015). Determinants of } \\
\text { Electronic word of mouth (EWOM) influence on hotel customers' purchasing decision. } \\
\text { Journal of Faculty of Tourism and Hotels, Fayoum University, 9(2/2), 194-223. }\end{array}$ & $\mathbf{X}$ & $\mathbf{X}$ & & \\
\hline $\begin{array}{l}\text { Adjei, M. T., Noble, S. M., \& Noble, C. H. (2010). The influence of C2C communications } \\
\text { in online brand communities on customer purchase behavior. Journal of the Academy of } \\
\text { Marketing Science, 38(5), 634-653. https://doi.org/10.1007/s11747-009-0178-5 }\end{array}$ & $\mathbf{X}$ & & & \\
\hline $\begin{array}{l}\text { Alonso, A. D., y Ogle, A. (2008). Importance of design for small Western Australian } \\
\text { wineries. Journal of Retail y Leisure Property, } 7(2), 139-147 .\end{array}$ & $\mathbf{X}$ & & & \\
\hline $\begin{array}{l}\text { Ayuso, M. (2017). Este vino está dedicado a los convictos que fundaron Australia (y los } \\
\text { conocerás por la realidad aumentada). https://www.directoalpaladar.com/nuevas- } \\
\text { tendencias/este-vino-esta-dedicado-a-los-convictos-que-fundaron-australia-y-los- } \\
\text { conoceras-por-la-realidad-aumentada (último acceso } 25 / 05 / 2020 \text { ) }\end{array}$ & & & $\mathbf{X}$ & $\mathbf{X}$ \\
\hline $\begin{array}{l}\text { Back, R M., "An Investigation into the Effect of Consumer Experience Tourism on Brand } \\
\text { Loyalty and Purchasing Behavior" (2015). Doctoral Dissertations. } 423 .\end{array}$ & $\mathbf{X}$ & $\mathbf{X}$ & & \\
\hline $\begin{array}{l}\text { Bethapudi, A. (2013). the Role of Ict in Tourism Industry. Journal of Applied Economics } \\
\text { and Business, 1(4),67-79. }\end{array}$ & $\mathbf{X}$ & $\mathbf{X}$ & & \\
\hline $\begin{array}{l}\text { Brochado, A., Stoleriu, O., y Lupu, C. (2019). Wine tourism: a multisensory experience. } \\
\text { Current Issues in Tourism, 0(0), 1-19. }\end{array}$ & $\mathbf{X}$ & $\mathbf{X}$ & $\mathbf{X}$ & \\
\hline $\begin{array}{l}\text { Bruwer, J., y Alant, K. (2009). The hedonic nature of wine tourism consumption: An } \\
\text { experiential view. International Journal of Wine Business Research, 21(3), 235-257. }\end{array}$ & $\mathbf{X}$ & & & \\
\hline $\begin{array}{l}\text { Bruwer, J., Gross, M. J., y Lee, H. C. (2016). Tourism destination image (TDI) perception } \\
\text { within a regional winescape context. Tourism Analysis, 21(2), 173-187. }\end{array}$ & $\mathbf{X}$ & $\mathbf{X}$ & & \\
\hline $\begin{array}{l}\text { Buhalis, D., y O'Connor, P. (2005). Information communication technology } \\
\text { revolutionizing tourism. Tourism Recreation Research, 30(3), 7-16. }\end{array}$ & $\mathbf{X}$ & $\mathbf{X}$ & $\mathbf{X}$ & \\
\hline $\begin{array}{l}\text { Carlsen, J., y Boksberger, P. (2015). Enhancing Consumer Value in Wine Tourism. Journal } \\
\text { of Hospitality and Tourism Research, 39(1), 132-144. }\end{array}$ & $\mathbf{X}$ & & & \\
\hline $\begin{array}{l}\text { Casaló, L. V., Flavián, C., e Ibáñez-Sánchez, S. (2017). Antecedents of consumer intention } \\
\text { to follow and recommend an Instagram account. Online Information Review. }\end{array}$ & & $\mathbf{X}$ & & \\
\hline $\begin{array}{l}\text { Canovi, M y Pucciarelli, F. (2019). Social media marketing in wine tourism: winery } \\
\text { owners'perceptions. Journal of Travel \& Tourims Marketing, } 36 \text { (6). }\end{array}$ & $\mathbf{X}$ & $\mathbf{X}$ & & \\
\hline
\end{tabular}


Cheong, R. (1995). The virtual threat to travel and tourism. Tourism Management.

Cho, M. H., y Sung, H. H. (2012). Travel Destination Websites: Cross-Cultural Effects on Perceived Information Value and Performance Evaluation. Journal of Travel and Tourism Marketing.

Choi, S., Lehto, X. Y., Morrison, A. M., y Jang, S. C. (2012). Structure of travel planning processes and information use patterns. Journal of Travel Research.

Chu, S. C., y Kim, Y. (2011). Determinants of consumer engagement in electronic WordOf-Mouth (eWOM) in social networking sites. International Journal of Advertising.

Crespi-Vallbona, M., y Mascarilla-Miró, O. (2020). Wine lovers: their interests in tourist experiences. International Journal of Culture, Tourism, and Hospitality Research, 14(2), 239-258.

Cristofol, FJ. Zamarreño-Aramendia, G, de-San-Eugenio;J. (2020). Effects of Social Media on Enotourism. Two Cases Study: Okanagan Valley (Canada) and Somontano (Spain). Sustainability, 12(17).

Davidson, R., y Keup, M. (2014). The Use of Web 2.0 as a Marketing Tool by European Convention Bureaux. Scandinavian Journal of Hospitality and Tourism.

Davies, M., Musango, J. K., \& Brent, A. C. (2016). A systems approach to understanding the effect of Facebook use on the quality of interpersonal communication. Technology in Society.

Dellarocas, C. (2003). The digitization of word of mouth: Promise and challenges of online feedback mechanisms. Management Science.

Dolan, R., y Goodman, S. (2017). Succeeding on social media: Exploring communication strategies for wine marketing. Journal of Hospitality and Tourism Management, 33, 23-30.

Dvoulety, O; Lukes, M; Misar, J (2020). Digital Communication of Wine Routes un Slovakia, Czech Republic, Germany and Austria. Proceedings of the $8^{\text {th }}$ international conference innovation management, entrepreneurship and sustainability (IMES 2020). 70-85.

Flavián, C., Ibáñez-Sánchez, S., y Orús, C. (2018). The impact of virtual, augmented and mixed reality technologies on the customer experience. Journal of Business Research, 100(January 2018), 547-560.

Gallace, A., Ngo, M. K., Sulaitis, J., y Spence, C. (2011). Multisensory presence in virtual reality: Possibilities y limitations. In Multiple Sensorial Media Advances and Applications: New Developments in MulSeMedia.

Geurin-Eagleman, A. N., \& Burch, L. M. (2016). Communicating via photographs: A gendered analysis of Olympic athletes' visual self-presentation on Instagram. Sport Management Review.

Gil-Rubio, Á., Jimenez-Barandalla, I. C., \& Mercado-Idoeta, C. (2017). Reputación corporativa online en la hotelería: el caso TripAdvisor. Esic Market Economic and Business Journal, 48(3), 595-608.

Godes, D.,y Mayzlin, D. (2004). Using online conversations to study word-of-mouth communication. Marketing science, 23(4), 545-560.

\begin{tabular}{|c|c|c|c|}
\hline & & $\mathbf{X}$ & \\
\hline $\mathbf{X}$ & & & \\
\hline $\mathbf{X}$ & $\mathbf{X}$ & & \\
\hline $\mathbf{X}$ & $\mathbf{X}$ & & \\
\hline $\mathbf{X}$ & $\mathbf{X}$ & & \\
\hline $\mathbf{X}$ & $\mathbf{X}$ & & \\
\hline \multirow[t]{2}{*}{$\mathbf{x}$} & $\mathbf{X}$ & & \\
\hline & $\mathbf{X}$ & & \\
\hline $\mathbf{X}$ & $\mathbf{X}$ & & \\
\hline $\mathbf{X}$ & $\mathbf{X}$ & & \\
\hline $\mathbf{X}$ & $\mathbf{X}$ & & \\
\hline \multirow[t]{3}{*}{$\mathbf{X}$} & $\mathbf{X}$ & $\mathbf{x}$ & $\mathbf{X}$ \\
\hline & & $\mathbf{X}$ & \\
\hline & $\mathbf{X}$ & & \\
\hline $\mathbf{X}$ & $\mathbf{X}$ & & \\
\hline $\mathbf{X}$ & $\mathbf{X}$ & & \\
\hline
\end{tabular}


Gonçalves, R., Martins, J., Pereira, J., Cota, M., y Branco, F. (2016). Promoting e-commerce software platforms adoption as a means to overcome domestic crises: The cases of portugal and spain approached from a focus-group perspective. Advances in Intelligent Systems and Computing.

Gretzel, U., Zhong, L., y Koo, C. (2016). Application of smart tourism to cities. International Journal of Tourism Cities.

Hall, C. M., y Mitchell, R. (2007). Wine marketing: A practical guide. In Wine Marketing: A Practical Guide.

Hennig-Thurau, T., Gwinner, K. P., Walsh, G., y Gremler, D. D. (2004). Electronic wordof-mouth via consumer-opinion platforms: What motivates consumers to articulate themselves on the Internet? Journal of Interactive Marketing.

Howell, R. T., y Guevarra, D. A. (2013). Buyinh Happiness: Differential Consumption Experiences for Material and Experiential Purchases (A. M. Columbus (Ed.); Issue July). Advances in Pshycology Research.

Huang, Y. C., Backman, S. J., Backman, K. F., y Moore, D. W. (2013). Exploring user acceptance of 3D virtual worlds in travel and tourism marketing. Tourism Management.

Huang, Y. C., Backman, K. F., Backman, S. J., y Chang, L. L. (2016). Exploring the Implications of Virtual Reality Technology in Tourism Marketing: An Integrated Research Framework. International Journal of Tourism Research.

Jalilvand, M. R., Esfahani, S. S., y Samiei, N. (2011). Electronic word-of-mouth: Challenges and opportunities. Procedia Computer Science, 3, 42-46.

Jeong, C., Holland, S., Jun, S. H., \& Gibson, H. (2012). Enhancing destination image through travel website information. International Journal of Tourism Research.

Jung, D., \& Cho, M.-H. (2015). A Discovery of the Positive Travel Experience in Pre-Trip, On-Site and Post-Trip Stage. Tourism Travel and Research Association: Advancing Tourism Research Globally., 1-6.

Karimi, S. (2013). A purchase decision-making process model of online consumers and its influential factor a cross sector analysis. In University of Manchester.

Költringer, C., y Dickinger, A. (2015). Analyzing destination branding and image from online sources: A web content mining approach. Journal of Business Research.

Kotler, P., Bowen, J. T., Makens, J.y Baloglu, S. (2017). Marketing for Hospitality and Tourism. Boston, MA: Pearson Education.

Ku, E. C. S., y Chen, C. Der., (2015) Cultivating travellers' revisit intention to e-tourism service: The moderating effect of website interactivity. Behaviour and Information Technology, 34(5), 465-478.

Ladhari, R., y Michaud, M. (2015). EWOM effects on hotel booking intentions, attitudes, trust, and website perceptions. International Journal of Hospitality Management.

Laverie, D. A., Humphrey, W. F., Velikova, N., Dodd, T. H., y Wilcox, J. B. (2011). Building wine brand communities with the use of social media: A conceptual model. In Proceedings of the 6th AWBR International Conference (pp. 9-10).

\begin{tabular}{|c|c|c|}
\hline $\mathbf{X}$ & & \\
\hline $\mathbf{X}$ & $\mathbf{X}$ & \\
\hline $\mathbf{X}$ & & \\
\hline $\mathbf{X}$ & & \\
\hline $\mathbf{X}$ & & \\
\hline $\mathbf{X}$ & $\mathbf{X}$ & \\
\hline $\mathbf{X}$ & & \\
\hline $\mathbf{X}$ & $\mathbf{X}$ & \\
\hline $\mathbf{X}$ & $\mathbf{X}$ & \\
\hline & & \\
\hline & & \\
\hline & & \\
\hline & & \\
\hline & & \\
\hline & & \\
\hline & & \\
\hline & & \\
\hline & & \\
\hline & & \\
\hline
\end{tabular}


Law, R., Buhalis, D., y Cobanoglu, C. (2014). Progress on information and communication technologies in hospitality and tourism. International Journal of Contemporary Hospitality Management.

Lewis, D. y Bridger D., (2001) The soul of the new consumer: Authenticity - What we buy and why in the new economy. Nicholas Brealey Publishing.

Litvin, S. W., Goldsmith, R. E., y Pan, B. (2008). Electronic word-of-mouth in hospitality and tourism management. Tourism Management.

Llodrà-Riera, I., Martínez-Ruiz, M. P., Jiménez-Zarco, A. I., y Izquierdo-Yusta, A. (2015). A multidimensional analysis of the information sources construct and its relevance for destination image formation. Tourism Management.

Lu, W., y Stepchenkova, S. (2012). Ecotourism experiences reported online: Classification of satisfaction attributes. Tourism Management.

Mak, A. H. N. (2017). Online destination image: Comparing national tourism organisation's and tourists' perspectives. Tourism Management.

Marasco, A., Buonincontri, P., van Niekerk, M., Orlowski, M., y Okumus, F. (2018).

Exploring the role of next-generation virtual technologies in destination marketing. Journal of Destination Marketing and Management.

Mariani, M. M., Di Felice, M., y Mura, M. (2016). Facebook as a destination marketing tool: Evidence from Italian regional Destination Management Organizations. Tourism Management.

Mário, C., \& Tânia, R. (2011). Emarketing for Wine Tourism. Enlightening Tourism. A Pathmaking Journal, 1(1), 93-110.

Martins, J., Gonçalves, R., Branco, F., Barbosa, L., Melo, M., y Bessa, M. (2017). A multisensory virtual experience model for thematic tourism: A Port wine tourism application proposal. Journal of Destination Marketing and Management.

Meng, F., y Xu, Y. (2012). Tourism shopping behavior: Planned, impulsive, or experiential? International Journal of Culture, Tourism and Hospitality Research, 6(3), 250-265.

Molinillo, S., Liébana-Cabanillas, F., Anaya-Sánchez, R., y Buhalis, D. (2018). DMO online platforms: Image and intention to visit. Tourism Management, 65, 116-130.

Perry Hobson, J. S., y Williams, A. P. (1995). Virtual reality: A new horizon for the tourism industry. Journal of Vacation Marketing.

Relevent, Case Studies: Teleporter. (2014). https://www.relevent.com/work/marriott/ teleporter (Último acceso 06/05/2020)

Rodríguez-Molina, M. A., Frías-Jamilena, D. M., y Castañeda-García, J. A. (2015). The contribution of website design to the generation of tourist destination image: The moderating effect of involvement. Tourism Management.

Santos, V. R., Ramos, P., Almeida, N., y Santos-Pavón, E. (2019). Wine and wine tourism experience: a theoretical and conceptual review. Worldwide Hospitality and Tourism Themes, 11(6), 718-730.

Senecal, S., y Nantel, J. (2004). The influence of online product recommendations on consumers' online choices. Journal of Retailing.

\begin{tabular}{|l|l|l|l|}
\hline $\mathbf{X}$ & $\mathbf{X}$ & \\
\hline $\mathbf{X}$ & & \\
\hline $\mathbf{X}$ & $\mathbf{X}$ & & \\
\hline $\mathbf{X}$ & $\mathbf{X}$ & & \\
\hline $\mathbf{X}$ & $\mathbf{X}$ & & \\
\hline $\mathbf{X}$ & $\mathbf{X}$ & \\
\hline $\mathbf{X}$ & $\mathbf{X}$ & & \\
\hline $\mathbf{X}$ & $\mathbf{X}$ & & \\
\hline & $\mathbf{X}$ & & \\
\hline & $\mathbf{X}$ & & \\
\hline & & & \\
\hline & & & \\
\hline & & & \\
\hline & & & \\
\hline & & & \\
\hline & & & \\
\hline & & & \\
\hline & & & \\
\hline & & & \\
\hline & & & \\
\hline & & & \\
\hline & & & \\
\hline
\end{tabular}




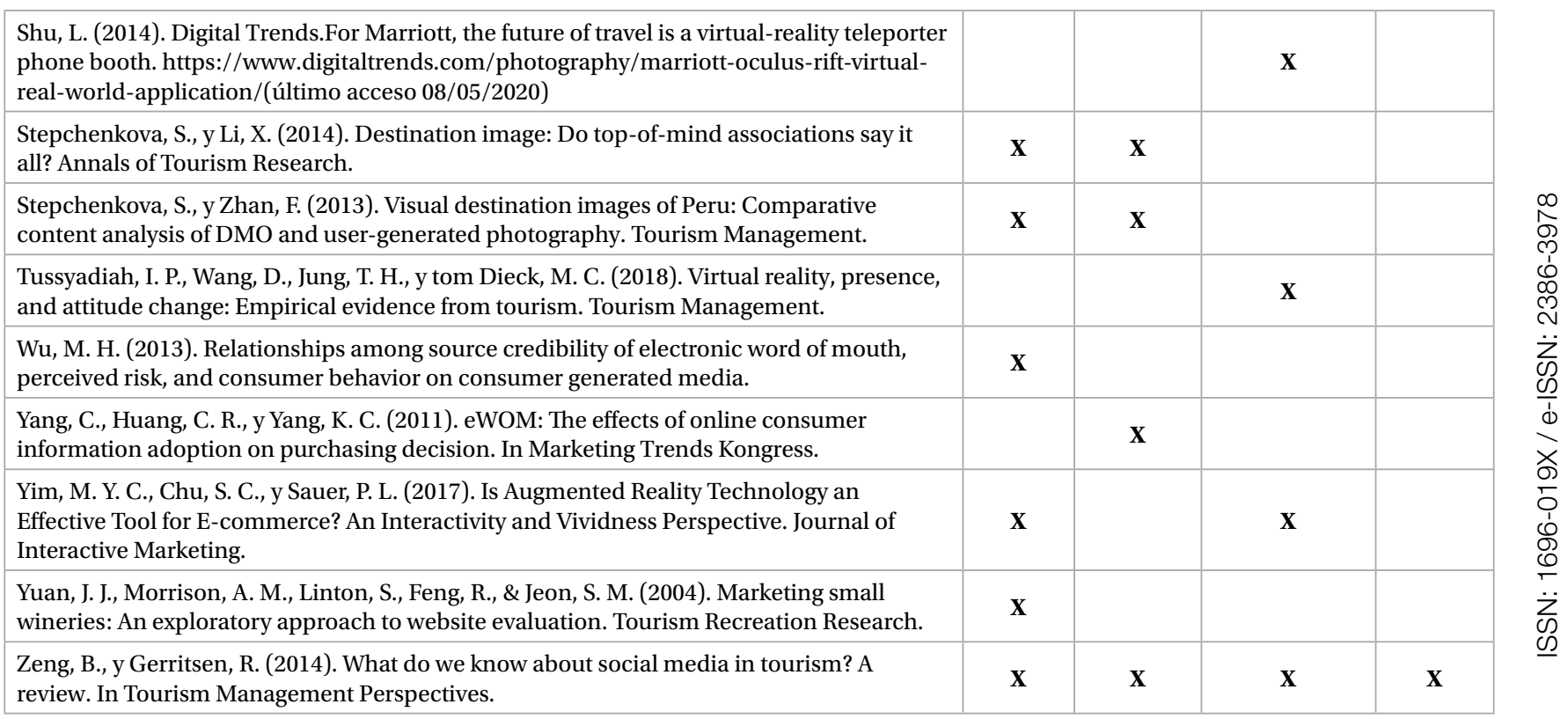

Fuente: elaboración propia en base a revisión de la literatura

\section{Enoturismo y rutas del vino. Una oportunidad de negocio}

El desarrollo de nuevas modalidades turísticas más allá del tradicional periodo vacacional de descanso, ha favorecido la creación y difusión de nuevos modelos turísticos que tienen como ejes los entornos naturales y el disfrute de las tradiciones locales (Cavicchi, 2013; Khartishvili, Muhar, Dax, Khelashvili, 2019; Privitera, 2010).

El turismo del vino contempla el interés de los viajeros por realizar desplazamientos y estancias para conocer los paisajes donde se producen los vinos, así como a realizar actividades que incrementen el conocimiento sobre este producto, procurando el progreso en las zonas vitivinícolas (Elias Pastor, 2006). Por tanto, se ha convertido en uno de los productos turísticos que con más celeridad ha sabido ajustarse al panorama turístico de las últimas décadas.

El enoturismo se ha desarrollado a lo largo de todo el mundo. En Europa en países como Francia, Italia, España, Portugal, Grecia, Alemania, Serbia, Rumanía o Georgia (Coros, Pop, y Popa, 2019; Dougherty, 2012; Festa, Shams, Metallo, y Cuomo, 2020; Khartishvili et al., 2019; Koch, Martin, Nash, 2013; López-Guzmán, García y Rodríguez, 2013; Lourenço-Gomes, Pinto y Rebelo, 2015; Trišić, Štetić, Privitera y Nedelcu, 2020; Wolikow, 2014) y de manera más intensa en los EE.UU., Canadá, Sudáfrica y Nueva Zelanda (Bruwer, 2003; Hall, Sharples, Cambourne, Macionis, Hall, et al., 2019; Hall, Sharples, Cambourne, Macionis y Telfer, 2019; Kirkman, Strydom, Van Zyl, 2013) y en zonas emergentes (Hojman y Hunter-Jones, 2012; Schlüter y Norrild, 2015). 
Las rutas del vino se definen como aquellos circuitos que delimitan los itinerarios en una zona geográfica, estando debidamente señalizados todos los espacios que motivan al turista en su recorrido. Getz y Brown (2006) establecieron que el concepto de enoturismo se puede observar desde tres diferentes enfoques: como comportamiento del consumidor, como estrategia de desarrollo regional, y como oportunidad de las bodegas para comercializar sus productos (Sigala, 2014).

El aumento del número de enoturistas significa una oportunidad para que las bodegas diversifiquen su mercado. Estas deben ser capaces de ofertar una vivencia completa a niveles sensoriales (Santos, Ramos, Almeida y Santos-Pavón, 2019; Charters, y Pettigrew, 2005) en las tres etapas que la componen: la pre-visita, la visita y la post-visita o nostalgia (Hall, Longo, Mitchell y Johnson, G. 1996).

Diseñar una experiencia enoturística, no es sencillo, puede ser entendida desde cuatro perspectivas diferentes (Getz y Brown, 2006b; Mănilă, 2012): la gastronómica (Espejel, Fandos y Flavián, 2011; Millán Vázquez De La Torre, Morales Fernández, y Pérez Naranjo 2014), la cultural (Getz et al., 2006; Williams y Kelly, 2001), la de patrimonio natural o terruño (Hall y Mitchell, 2002; Correia y Brito 2016) y la educativa (Buhalis y O'Connor, 2005).

La pluralidad de ofertas siempre debe diseñarse en torno a la atracción principal que es el vino, asumido como una bebida de moda y ligado al consumo de una experiencia hedonista (Bruwer, y Alant, 2009), lo que convierte al enoturismo en una experiencia vital.

La búsqueda de placer como principal motivación a la hora de realizar el viaje, hace que el enoturista sea un turista más exigente en términos de calidad, personalización y diferenciación (Espejel et al., 2011). Además, es un cliente que valora mucho su tiempo y busca un crecimiento personal con la visita (Buhalis y O'Connor, 2005) por ello se puede decir que también busca satisfacer necesidades epistemológicas (Charters, y Ali-Knight, 2002).

El alto grado de exigencia del enoturista se ha convertido en uno de los mayores retos para las bodegas, pero también es una gran oportunidad, ya que el turista del vino al quedar satisfecho con la experiencia la recomienda en mayor medida que el turista al uso (Ruiz-Romero de la Cruz, Zamarreño-Aramendia y Cruz-Ruiz, 2020), debido a su carácter sociable (Laverie, Humphrey, Velikova, Dodd, y Wilcox, 2011), cerca de un 95\% de los visitantes de bodegas o viñedos lo hacen acompañados (Bruwer, y Reilly, 2006). Según los datos recogidos por ACEVIN (2019) el nivel de satisfacción de los enoturistas se sitúa en 8,56 puntos sobre 10 y un $99,4 \%$ de los visitantes recomendaría la visita.

\section{Las etapas de la visita y la revisión de la literatura}

La comunicación juega un papel primordial en la configuración de la experiencia del enoturista ya que el vino es un producto holístico (Cristofol, Zamarreño-Aramendia, De-San-Eugenio, 2020). Los diferentes canales digitales se complementan para asegurar y afianzar la experiencia que el visitante tiene in situ. Las etapas de la visita han sido definidas por diversos autores de los que cabe destacar a Hall y Macionis (1998), Hall et al (2006), Fuentes, Moreno-Gil, González y Ritche (2015), Rodríguez Zulaica (2016); o Fernández-Cavia, Vinyals-Mirabent, Fernández-Planells, Weber, y Pedraza-Jiménez (2020), entre otros. 


\subsection{La etapa pre-visita}

En un principio la etapa pre-visita se inicia por un deseo y no una necesidad, pues el enoturista está guiado por impulsos hedonistas (Holbrook, y Hirschman, 1982). Aquí la imaginación es la encargada de generar las expectativas de la visita, junto al estudio de experiencias previas de otros consumidores (Carù, y Cova, 2003). Las empresas deben centrarse en superar estas expectativas en la etapa de experiencia in situ, para imponerse como la opción preferida frente a sus competidores. Para ello, deben tener en cuenta que el cliente ha de sentirse involucrado en la experiencia, sorprendido por la novedad, debiendo aportarle conocimientos sobre la cultura del vino y su entorno, la conjunción de todas estas particularidades conseguirá generar la sensación de nostalgia en la etapa post-compra (Crespi-Vallbona, y Mascarilla-Miró, 2020).

Para la búsqueda de la información el enoturista prefiere acudir a canales informales y externos a la propia bodega, donde el contenido este generado por visitantes previos (Wu, 2013). La importancia de que el E-WOM (boca a boca digital) gane terreno al WOM (boca a boca) reside en el carácter público y abierto del E-WOM lo que le permite a la bodega controlar lo que otros dicen de su marca y de su experiencia, para poder ofrecer una orientación centralizada en el consumidor (Dellarocas, 2003; Jalilvand, Esfahani, y Samiei, 2011). Esta búsqueda de información debe estar apoyada en el mundo digital a través del uso de las páginas web, que en la mayoría de casos suponen el primer contacto del cliente con la bodega y sus vinos (Cho, y Sung, 2012).

Cualquier destino turístico debe disponer de un dominio Web (Jeong, Holland, Jun, y Gibson, 2012), el uso correcto de este canal de comunicación genera una imagen positiva y aumenta la intención de visita Llodrà-Riera et al. (2015). En ella deben existir enlaces a las redes sociales de la bodega, que fomenten la participación del cliente en esas plataformas (Davidson y Keup, 2014).

Las redes sociales son indispensables para compartir contenido audiovisual de la bodega e interactuar de una manera más directa con el cliente. De este modo, se produce un aumento de la conversación en la red (Molinillo, Liébana-Cabanillas, Anaya-Sánchez, y Buhalis, 2018) y se facilita la creación de una comunidad de marca (Zeng, y Gerritsen, 2014). En línea con esta estrategia de marketing, hoy en día el uso del "marketing de influyente" es indispensable, pues tiene una gran influencia en la decisión de compra (Yang, Huang y Yang, 2011). Se trata de una promoción mucho más informal y directa, desde la 'voz' de la experiencia, lo cual trasmite mayor confianza que los medios de promoción tradicionales.

Los consumidores se involucrarán de una manera más intensa cuanto mayor sea el nivel de participación en la creación de contenido, y por tanto el grado de confianza en la información ofrecida por parte del cliente potencial aumentará, pues como señala $\mathrm{Wu}$ (2013) el cliente potencial tiene más en cuenta las opiniones de amigos o referentes en el mundo del vino, que la propia información ofrecida por la bodega. Como indica Molinillo et al. (2018), las bodegas necesariamente deben estar presentes en las redes sociales más populares: Instagram, Youtube y Facebook, cuyas características para la creación de ventajas se recogen en la Tabla 2. 
Tabla 2. Redes sociales y sus características en relación con el enoturismo

\begin{tabular}{|c|c|}
\hline Red Social & Características en torno al enoturismo \\
\hline Instagram & $\begin{array}{l}\text { - Instagram fue diseñada para compartir fotos y videos cortos, permite la interacción con otros usuarios, de } \\
\text { manera pública, a través de comentarios y "me gustas" y de forma privada a través de mensajes privados } \\
\text { (Geurin-Eagleman y Burch 2016). } \\
\text { - Nuevos formatos para compartir contenido: historias caracterizadas por videos o fotografías de } 15 \text { segundos } \\
\text { que desaparecen a las } 24 \text { horas, IGTV fuerte competidor de Youtube y por último videos en streaming. Se } \\
\text { prevé que la herramienta de Stories genere } 7.5 \text { mil millones de dólares en } 2021 \text { (Fondevilla-Gascón et al., } \\
2020 \text { ). } \\
\text { - Esta red social es la que, presenta mayores diferencias en el proceso de creación de imagen de destino en } \\
\text { comparación con los sitios web. No requiere un gran nivel de implicación por parte del consumidor, pues } \\
\text { navegar e interactuar en ella es sencillo. Presenta los valores más altos en intención de visita tras la consulta } \\
\text { y en incremento de la imagen general del destino, representando el } 50 \% \text { de la desviación de la intención de la } \\
\text { visita del estudio. Es la mejor plataforma tanto para construir la imagen de destino como para captar turistas } \\
\text { (Fantani y Suyadnya, 2015). }\end{array}$ \\
\hline Facebook & $\begin{array}{l}\text { - Facebook permite la creación de perfiles públicos, tanto personales como empresariales, en ellos se pueden } \\
\text { cargar información personal, de contacto, etc. Conecta a usuarios, organizaciones y marcas, pudiendo com- } \\
\text { partir todo tipo de documentos audiovisuales e interactuar con ellos tanto de manera pública como privada } \\
\text { (Davies, Musango y Brent, 2016). } \\
\text { - Requiere un alto nivel de implicación por parte del turista, es decir, presenta una mayor motivación en rela- } \\
\text { ción con la empresa, lo que influencia de manera positiva la imagen de destino. La influencia positiva en la } \\
\text { intención de visita es menor que la presentada por Instagram o el propio sitio web. Incluso Youtube explica } \\
\text { más la desviación en la intención de la visita que esta plataforma. }\end{array}$ \\
\hline Youtube & $\begin{array}{l}\text { - Youtube es la plataforma mundialmente más utilizada para compartir videos, además permite interactuar a } \\
\text { través de comentarios y “me gustas” (Smith, Fischer y Chen, 2012). } \\
\text { - YouTube ha introducido la novedad de chats en directo en los que los creadores interactúan en momento } \\
\text { en el que el video es estrenado, en búsqueda de una interacción más directa debido a la pérdida de cuota de } \\
\text { mercado a favor de otro tipo de plataformas como Instagram Videos (IGTV). } \\
\text { - Es la plataforma con menos nivel de implicación por parte del usuario de las tres analizadas, lo que provoca } \\
\text { que su influencia sobre la creación de la imagen de destino sea prácticamente inexistente y la más baja en } \\
\text { comparación con las demás plataformas donde se incluye el sitio web. Este patrón se repite respecto a la } \\
\text { influencia en la intención de visita, por lo que queda demostrado que es la plataforma menos atractiva a la } \\
\text { hora de atraer clientes. }\end{array}$ \\
\hline
\end{tabular}

Fuente: elaboración propia en base a la revisión de la literatura

La experiencia enoturística al estar tan influenciada por los sentidos (Getz, 2000), hace que cada turista la viva de una manera totalmente diferente (Cho et al., 2017), lo que hace aumentar el riesgo de que existan desajustes entre las expectativas generadas por el consumidor en esta fase previa de elección y lo realmente vivido durante la visita (Carlsen, y Boksberger, 2015). Para acabar con este inconveniente, cuanto mayor sea la similitud entre la información recibida en esta fase y la realidad, mayor será la satisfacción del enoturista (Ku, y Chen, 2015). Para conseguir este acercamiento a la realidad y disponer de una información más detallada de la experiencia in situ este modelo plantea el uso de la realidad aumentada y la realidad virtual (Marasco, Buonincontri, Van Niekerk, Orlowski, y Okumus, 2018). La combinación de todos estos recursos digitales permite que el turista genere un fuerte 
vínculo emocional con el destino (Huang, Backman, Backman, y Moore, 2013). Y al tratarse de una experiencia 'Prueba antes de comprar' (Tussyadiah, Wang, Jung, y Dieck, 2018) aumenta mucho la intención de visita (Marasco et al., 2018).

\subsection{La experiencia in situ}

En esta fase es donde aparecen las sensaciones de satisfacción o disgusto en relación a la visita enoturística y por tanto respecto a la marca (Howell, y Guevarra, 2013). Sin embargo, existen pocos estudios que hayan medido el impacto de la experiencia in situ en el comportamiento del comprador. A pesar de ello, la literatura académica coincide en que el éxito de la creación de experiencias memorables, depende de la capacidad de la bodega para generar impactos únicos que superen las expectativas de los enoturista. Vivencias que sean capaces de generar un E-WOM positivo y la posible repetición del cliente (Hosany y Witham, 2010; Fernandes et al., 2016)

Para poder responder a las necesidades del cliente en su visita, es necesario identificar al turista del vino a través de la segmentación del mercado (Alebaki, y Iakovidou, O. 2011; Cho, Bonn, Brymer, 2017; Bruwer et al., 2009). El estudio de Cho et al., (2017) hace una distinción de los enoturistas en cinco segmentos atendiendo a las limitaciones que encuentran los turistas potenciales a la hora de tomar la decisión de visita. De estos cinco segmentos se destacan dos como los más interesantes a la hora de dirigir la creación de la experiencia. Por un lado, el segmento de aquellos que encuentran mínimas limitaciones y, por lo tanto, se sienten muy atraídos por la idea de realizar la visita. El único condicionamiento que señala este grupo es la falta de tiempo y dinero, aunque en una medida muy por debajo de la media del resto de los grupos. El otro segmento interesante es el de las familias, que presenta todavía menores restricciones respecto al tiempo y el dinero, lo que indica que su capacidad económica es superior a la media, el principal factor limitante señalado por este grupo es el de la falta de planes o actividades para las familias en las visitas.

Otra peculiaridad que presentan ambos segmentos es que suelen ser clientes que ya han realizado visitas similares y que presentan intenciones superiores a la media, tanto en la predisposición a realizar actividades enoturísticas, a pagar un precio extra por ellas y a recomendarlas tras una experiencia positiva. Estas características son claves en la diferenciación del enoturista del turista tradicional de "sol y playa", el enoturista se trata de un turista experimentado y con conocimientos sobre el vino por ello es mucho más exigente en los servicios que desea recibir (Brown, y Getz, 2005)

El turista del vino al visitar la bodega busca conocer la relación del viñedo, el vino y el entorno rural que le rodea, desea aprender más sobre el producto, y también siente la curiosidad por conocer la región y sus gentes (Hall y Mitchel, 2007). Así podemos hablar del turismo educativo motivado por situaciones donde la experiencia nace de forma espontánea, auténtica y aporta novedad respecto a la forma de presentar las experiencias que tienen otras bodegas (Jung y Cho, 2015).

Haciendo uso de códigos QR para ampliar la información del tour tradicional o creando nuevas experiencias a través del uso de la tecnología CAVE que consiste en una habitación donde el entorno virtual es proyectado sobre las paredes, techo o suelo según este programada (Martins et al., 2017).

Estas tecnologías no son caras e incluso pueden ahorrar costes de personal y además las familias que viajen con menores se ven se divierten con los medios digitales generando mayor satisfacción en la visita. 
En esta etapa el contenido de la experiencia es muy similar al que ya se ofrecía, pero el método por el cual se acerca al consumidor, es el que aporta la gran novedad que el cliente busca para construir una experiencia positiva y memorable (Flavián et al., 2018; Tussyadiah et al., 2018).

\subsection{Experiencia post-compra o de nostalgia}

La memoria juega un papel fundamental una vez finaliza el viaje, pues es el elemento principal de la reconstrucción de las sensaciones vividas en la experiencia in situ. Los recuerdos tienden a generar sensaciones inciertas engrandeciendo las experiencias positivas y quitándole valor a las negativas (Mitchell, Thompson, Peterson y Cronk, 1997).

Hoy en día cualquiera puede convertirse en fotógrafo con su smartphone, lo que facilita el proceso de rememoración. Hasta hace poco, este proceso solo era posible a través de la imaginación, comportándose como recordatorio de la experiencia vivida (Swanson, 2004). El hecho de introducir imágenes que deben estar tomadas en distintos puntos, favorece el desarrollo artístico y personal del cliente y al mismo tiempo beneficia a la bodega, pues en multitud de ocasiones las imágenes tomadas por los clientes comunican un mensaje que la bodega no ha sabido reflejar o que al no ver con ojos de turista no ha valorado como interesante (Stepchenkova y Zhan, 2013).

En esta etapa los clientes deben de dejar de ser simples espectadores y convertirse en fans, que son clientes fieles y con mayor tendencia a la repetición de la visita y la recomendación a amigos y familiares (Dodd, 1999). Es muy importante que las redes se conviertan en una comunidad que aporte valor y que no solo se base en contenido promocional, pues debe incitar al cliente a dar su opinión libremente sobre la marca y ayudarlo a conectar con otros seguidores de esta y con la propia bodega (Dolan et al., 2017)

\subsection{Las nuevas tecnologías y el turismo de experiencias del vino. Algunos ejemplos}

Las bodegas no han permanecido ajenas a los cambios y posibilidades que ofrecía la tecnología, para ofertar una experiencia diferenciadora y enriquecedora para el enoturista en la pre-visita y en la visita, de modo que supongan un acicate para las tradicionales degustaciones de vino en las instalaciones bodegueras, explorando de este modo, nuevas necesidades de los enoturistas (Getz, 2000; Pasternak, 2004; Garibaldi y Pozzi, 2018). La tecnología más utilizada es aquella que aprovecha el potencial que ofrece la realidad virtual (Garibaldi y Sfodera, 2020), ofreciendo enormes posibilidades, tanto en el ámbito de la educación y el aprendizaje, como en el campo de las aplicaciones lúdicas, siendo especialmente útil en entornos de difícil acceso (Guttentag, 2010).

En el caso español, se han analizado dos bodegas que han apostado por este tipo de tecnología: Bodegas Ramón Bilbao y Bodegas Gandía. La primera, localizada en la localidad riojana de Haro, ha realizado un esfuerzo para desarrollar productos y servicios innovadores que atraigan al enoturista. De este modo los visitantes pueden hacer uso de la tecnología "Oculus Rift”, fundamentada en la realidad virtual de manera que permite al enoturista conocer el proceso de elaboración del vino al completo. Esta tecnología se aprovecha para dar a conocer los productos que más tarde se pueden catar, ofreciendo una experiencia audiovisual que traspasa las barreras físicas donde el vino y el paisaje del vino son los protagonistas. A esta experiencia se le añade el valor formativo, ya que permite que el visitante conozca la historia y valores de la bodega y el entorno que la rodea (bodegasramonbilbao.es 2018). 
Las valencianas Bodegas Gandía, junto con su distribuidor en Holanda, optaron en 2017 por ofrecer una experiencia de realidad virtual en el Samsung Galaxy Studio de Amsterdam, a través del dispositivo Samsung Gear VR. El objetivo era acercar las bodegas a los futuros clientes despertando su interés en la fase de pre-visita, ofreciendo una cata en su finca de Hoya de las Cadenas donde se encuentra su centro enoturístico. La tecnología permite eliminar las barreras que imponen la distancia y disfrutar de seis vinos seleccionados, así como de su historia y peculiaridades. La propia bodega define la experiencia como un modo de acercar la enología al consumidor final, de una manera innovadora, rompiendo con los códigos tradicionales imperantes en el mundo del vino.

En las antípodas dos casos merecen ser mencionados. En primer lugar, las bodegas australianas Seppeltsfield, que cuentan con una historia de 165 años. En este caso, los usuarios utilizan sus propios teléfonos móviles acompañados de gafas de realidad virtual para realizar un recorrido por la finca de Barossa Valley, de modo que los enoturistas tienen la oportunidad de disfrutar de panorámicas que les muestran el arte, la gastronomía y el vino sumergidos en los terrenos de la bodega. Como complemento los visitantes a su sitio web pueden disfrutar de recorridos en $360^{\circ}$ de sus instalaciones. Además, las posibilidades de acceso al recorrido también se puede realizar a través de un enlace al sitio web o mediante el escaneo de un código de respuesta rápida (QR), que Seppeltsfield ha comenzado a incluir en las etiquetas de sus vinos (seppeltsfield.com.au 2020).

En Nueva Zelanda encontramos Brancott Estate, una bodega con más de 40 años elaborando caldos, que destaca por su compromiso con la innovación y la sostenibilidad en la elaboración de vinos. En 2016 puso en marcha "Red Shed Experience", utilizando la realidad virtual para darse a conocer en sus eventos corporativos. Mediante el uso de un dispositivo de realidad virtual, los usuarios pueden visitar los viñedos y bodega, descubriendo la maduración y el proceso de elaboración del vino. En este caso, se utilizan dispositivos específicos que liberan aromas de uvas y frutas que ayudan a que los enoturistas tengan una experiencia inmersiva completa (brancottestate.com 2018).

\section{Conclusiones}

La revisión de la literatura refleja la velocidad de los cambios que afectan a la comunicación digital y más concretamente al turismo del vino. Si las bodegas quieren adaptarse a la nueva realidad del mercado, deben modificar su modelo de negocio digitalizando todas las fases del proceso de visita. En la figura 1 se resume cada una de las fases mencionadas, donde se aprecia su capacidad de llegar a nuevos segmentos, consolidando los anteriores, ofreciendo novedad, satisfacción, lo que redunda en un aumento de la lealtad de los visitantes y consumidores potenciales. 
Figura 1: Digitalización y fases de la visita enoturística

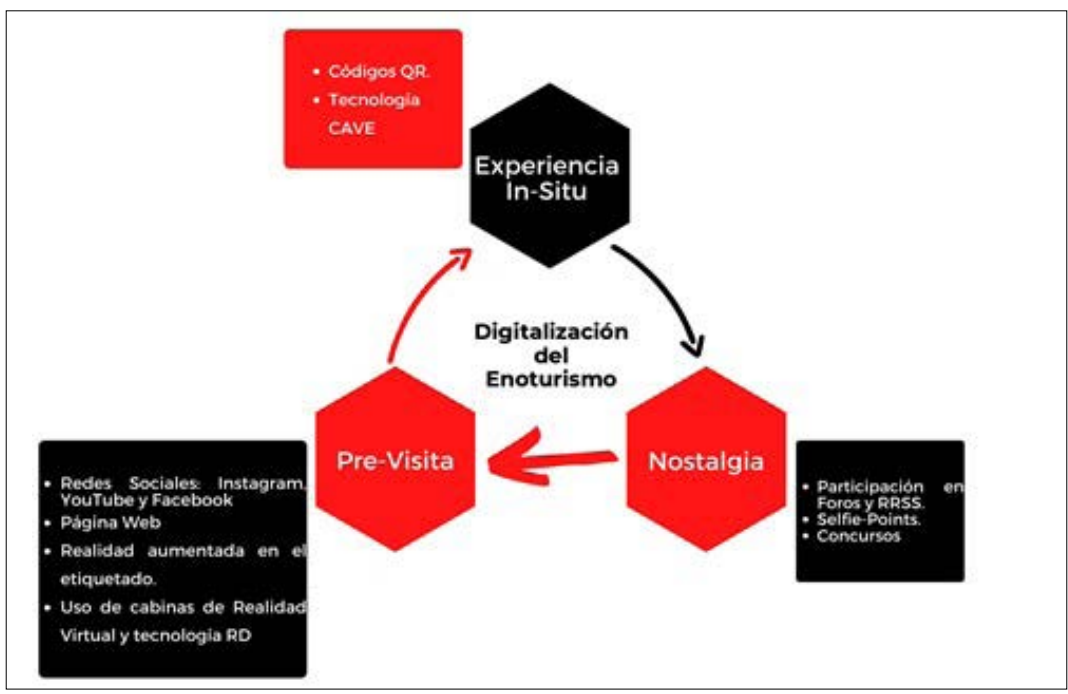

Fuente: elaboración propia en base a la revisión de la literatura

Hoy en día debemos hablar del turismo inteligente como el futuro del mercado (Shen, S., Sotiriadisforajidos, y Zhang, 2020) y por ello las bodegas no deben quedar al margen de la ola de renovación y adaptación, o correrán el riesgo de que su reclamo como destino turístico nunca llegue a posicionarse en el nivel de otros competidores. El uso de los recursos digitales para instruir al enoturista aporta la novedad, autenticidad y espontaneidad que necesitan para generar y reforzar sus sentimientos positivos hacia la bodega.

A lo largo de la revisión de la literatura se ha comprobado que en la actualidad las tecnologías han evolucionado de manera que pueden ofrecer soluciones en las que los usuarios puedan participar virtualmente de experiencias inmersivas cada vez más realistas. Baus y Bouchard (2016) y Delplanque et al. (2008), señalan las grandes posibilidades de desarrollar experiencias multisensoriales gracias a la tecnología. La interacción de los usuarios de las webs relacionadas con el mundo del vino debe convertirse en una experiencia lo más inmersiva posible. Un gran número de bodegas han adoptado e implementado sitios webs, que combinan diferentes herramientas de redes sociales, cubriendo el proceso de toma de decisiones, desde la búsqueda de información inicial hasta la comunicación previa y posterior con los potenciales consumidores y enoturistas (Canovi y Pucciarelli, 2019).

En diversos sentidos, el eWOM muestra muchas características del consumidor turístico moderno. La capacidad para obtener información y opinión en tiempo real y convertirla en datos utilizables en un futuro más o menos inmediato, pone de manifiesto la creciente importancia del uso de las TICs para reconocer y mejorar la experiencia turística eliminando las barreas geográficas y sociales. De este modo, la utilización de los diferentes recursos digitales suponen una ventaja, que pueden añadir un mayor conocimiento de las bodegas y de los vinos comercializados. En la actualidad, las bodegas se benefician de una manera muy limitada de todas las posibilidades. 
En el mundo digital de las bodegas la falta de desarrollo de campañas de marketing implica la pérdida de oportunidades para consolidar a los clientes actuales y alcanzar una nueva demanda potencial, que redundaría en un turismo más sostenible. Por otro lado, se están desaprovechando las ventajas asociadas al carácter público del E-WOM, en contraste con la privacidad del WOM, lo que permite ofrecer un producto mucho más orientado al cliente, solucionar y dar respuesta a los desajustes encontrados por parte del cliente entre expectativa y realidad.

Se han detectado diversos vacíos, que deberían tenerse en cuenta como nuevas vías de investigación en el desarrollo del enoturismo, tanto desde el punto de vista teórico como práctico. Se conoce el perfil del turista del vino, cómo se relaciona con su entorno, cómo interactúa, como busca vivir una experiencia diferenciadora y cómo expresa su grado de satisfacción. A pesar de que toda esta información es de gran valor para el proceso de digitalización, se detecta la falta de investigaciones sobre la experiencia digital ligada al enoturismo o su adaptación al concepto de turismo inteligente.

\section{Agradecimientos}

Este artículo ha sido traducido por Lucía Otaola Puche.

\section{Referencias bibliográficas}

Abd-Elaziz, M. E., Aziz, W. M., Khalifa, G. S., Abdel-Aleem, M. (2015). Determinants of Electronic word of mouth (EWOM) influence on hotel customers' purchasing decision. Journal of Faculty of Tourism and Hotels, Fayoum University, 9(2/2), 194-223.

Adjei, M. T., Noble, S. M., \& Noble, C. H. (2010). The influence of C2C communications in online brand communities on customer purchase behavior. Journal of the Academy of Marketing Science, 38(5), 634-653. https://doi.org/10.1007/s11747-009-0178-5.

Alba, J. W., y Williams, E. F. (2013). Pleasure principles: A review of research on hedonic consumption. In Journal of Consumer Psychology. https://doi.org/10.1016/j.jcps.2012.07.003

Alebaki, M., y Iakovidou, O. (2011). Market segmentation in wine tourism: A comparison of approaches. Tourismos, 6(1), 123-140.

Ali-Knight, J., y Carlsen, J. (2003). An exploration of the use of 'extraordinary' experiences in wine tourism. In Proceedings of the International Colloquium in Wine Marketing (pp. 1-8). Wine marketing Group, University of South Australia Adelaide, Australia. https://pdfs.semanticscholar.org/673c/dcc1979b49a7b91948c235234c7dlfdefbbb.pdf

Akaka, M. A., Vargo, S. L., y Schau, H. J. (2015). The context of experience. Journal of Service Management. https://doi.org/10.1108/ JOSM-10-2014-0270

Alonso, A. D., y Ogle, A. (2008). Importance of design for small Western Australian wineries. Journal of Retail y Leisure Property, 7(2), 139-147. https://link.springer.com/article/10.1057/rlp.2008.6

Arnould, E. J., Price, L., y Zinkhan, G. M. (2002). Consumers: McGraw-Hill/Irwin.

Ayuso, M. (2017). Este vino está dedicado a los convictos que fundaron Australia (y los conocerás por la realidad aumentada). https://www.directoalpaladar.com/nuevas-tendencias/este-vino-esta-dedicado-a-los-convictos-que-fundaron-australia-y-losconoceras-por-la-realidad-aumentada (último acceso 25/05/2020) 
Back, R M., "An Investigation into the Effect of Consumer Experience Tourism on Brand Loyalty and Purchasing Behavior" (2015). Doctoral Dissertations. 423. https://scholarworks.umass.edu/cgi/viewcontent.cgi?article=1432\&context=dissertations_2

Baus, O., y Bouchard, S. (2016). Exposure to an unpleasant odour increases the sense ofpresence in virtual reality.Virtual Reality,1-16. http://dx.doi.org/10.1007/s10055-016-0299-3.

Beatty, S. E., y Ferrell, M. E. (1998). Impulse buying: Modeling its precursors. Journal of Retailing. https://doi.org/10.1016/ S0022-4359(99)80092-X

Beer, J., Fast Company.(2014). This Marriott Teleporter Will (Virtually) Take You To London And Maui. https://www.fastcompany. com/3036007/this-marriott-teleporter-will-virtually-take-you-to-london-and-maui (último acceso 05/05/2020)

Bethapudi, A. (2013). the Role of Ict in Tourism Industry. Journal of Applied Economics and Business, 1(4), 67-79. http://www. aebjournal.org/articles/0104/010406.pdf

Bloom, J., Kompier, M., Geurts, S., de Weerth, C., Taris, T., y Sonnentag, S. (2009). Do we recover from vacation? Meta-analysis of vacation effects on health and well-being. In Journal of Occupational Health. https://doi.org/10.1539/joh.K8004

Bolton, R. N., Gustafsson, A., McColl-Kennedy, J., Sirianni, N. J., y Tse, D. K. (2014). Small details that make big differences: A radical approach to consumption experience as a firm's differentiating strategy. Journal of Service Management. https://doi. org/10.1108/JOSM-01-2014-0034

Boote, D. N., y Beile, P. (2005). Scholars Before Researchers: On the Centrality of the Dissertation Literature Review in Research Preparation. Educational Researcher. https://doi.org/10.3102/0013189X034006003

Briner, R. B., y Denye, D. (2012). Systematic Review and Evidence Synthesis as a Practice and Scholarship Tool. In The Oxford Handbook of Evidence-Based Management.

Briner, R. B., y Walshe, N. D. (2014). From passively received wisdom to actively constructed knowledge: Teaching systematic review skills as a foundation of evidence-based management. In Academy of Management Learning and Education. https://doi. org/10.5465/amle.2013.0222

Brochado, A., Stoleriu, O., y Lupu, C. (2019). Wine tourism: a multisensory experience. Current Issues in Tourism, 0(0), 1-19. https://doi.org/10.1080/13683500.2019.1649373

Brown, G., y Getz, D. (2005). Linking wine preferences to the choice of wine tourism destinations. Journal of Travel Research. https://doi.org/10.1177/0047287504272027.

Bruwer, J. (2003). South African wine routes: Some perspectives on the wine tourism industry's structural dimensions and wine tourism product. Tourism Management. https://doi.org/10.1016/S0261-5177(02)00105-X

Bruwer, J.,y Reilly, M. (2006). The power of word-of-mouth communication as an information source for winery cellar door visits. Australian and New Zealand Wine Industry Journal, 21(3), 43-51. https://papers.ssrn.com/sol3/Delivery.cfm/SSRN_ID2226244_ code87814.pdf?abstractid=2226244\&mirid=1 
Bruwer, J., y Alant, K. (2009). The hedonic nature of wine tourism consumption: An experiential view. International Journal of Wine Business Research, 21(3), 235-257. https://www.emerald.com/insight/content/doi/10.1108/17511060910985962/full/ pdf?title=the-hedonic-nature-of-wine-tourism-consumption-an-experiential-view

Bruwer, J., Gross, M. J., y Lee, H. C. (2016). Tourism destination image (TDI) perception within a regional winescape context. Tourism Analysis, 21(2), 173-187. https://doi.org/10.3727/108354216X14559233984692

Bugeja, M., Dingli, A., \& Seychell, D. (2018). Selfie as a Motivational Tool for City Exploration. International Association for Development of the Information Society. http://files.eric.ed.gov/fulltext/ED590388.pdf

Buhalis, D. (1993). RICIRMS as a strategic tool for small and medium tourism en- terprises. Tourism Management, 14(5). https:// doi.org/10.1016/0261-5177(93)90005-6

Buhalis, D. (1998). Strategic use of information technologies in the tourism industry. Tourism Management, 19(5). https://doi. org/10.1016/S0261-5177(98)00038-7

Buhalis, D., Jafari, J., y Werthner, H. (1997). Information technology and the reengineering of tourism. Annals of Tourism Research. Buhalis, D., y O'Connor, P. (2005). Information communication technology revolutionizing tourism. Tourism Recreation Research, 30(3), 7-16. https://doi.org/10.1080/02508281.2005.11081482

Canovi, M., y Pucciarelli, F. (2019). Social media marketing in wine tourism: winery owners' perceptions. Journal of Travel \& Tourism Marketing, 36(6), 653-664. https://doi.org/10.1080/10548408.2019.1624241

Carlsen, J., y Boksberger, P. (2015). Enhancing Consumer Value in Wine Tourism. Journal of Hospitality and Tourism Research, 39(1), 132-144. https://doi.org/10.1177/1096348012471379

Carù, A., y Cova, B. (2003). Revisiting consumption experience: A more humble but complete view of the concept. Marketing Theory. Charters, S., y Ali-Knight, J. (2002). Who is the wine tourist? Tourism Management. 23(3), 311-319.

Casaló, L. V., Flavián, C., \& Ibáñez-Sánchez, S. (2017). Antecedents of consumer intention to follow and recommend an Instagram account. Online Information Review. https://doi.org/10.1108/OIR-09-2016-0253

Cavicchi, A. (2013). Preserving the authenticity of food and wine festivals: the case of Italy. Il Capitale Culturale: Studies on the Value of Cultural Heritage

Charters, S., y Pettigrew, S. (2005). Is wine consumption an aesthetic experience? In Journal of Wine Research. https://doi. org/10.1080/09571260500327663

Cheong, R. (1995). The virtual threat to travel and tourism. Tourism Management. https://doi.org/10.1016/0261-5177(95)00049-T

Cho, M. H., y Sung, H. H. (2012). Travel Destination Websites: Cross-Cultural Effects on Perceived Information Value and Performance Evaluation. Journal of Travel and Tourism Marketing. https://doi.org/10.1080/10548408.2012.666169

Cho, M., Bonn, M. A., y Brymer, R. A. (2017). A Constraint-Based Approach to Wine Tourism Market Segmentation. Journal of Hospitality and Tourism Research. https://doi.org/10.1177/1096348014538049 
Choi, S., Lehto, X. Y., Morrison, A. M., y Jang, S. C. (2012). Structure of travel planning processes and information use patterns. Journal of Travel Research. https://doi.org/10.1177/0047287510394191

Chu, S. C., y Kim, Y. (2011). Determinants of consumer engagement in electronic Word-Of-Mouth (eWOM) in social networking sites. International Journal of Advertising. https://doi.org/10.2501/IJA-30-1-047-075

Coros, M. M., Pop, A. M., \& Popa, A. I. (2019). Vineyards and wineries in Alba County, Romania towards sustainable business development. Sustainability https://doi.org/10.3390/su11154036

Correia R., Brito C. (2016) Wine Tourism and Regional Development. In: Peris-Ortiz M., Del Río Rama M., Rueda-Armengot C. (eds) Wine and Tourism. Springer, Cham. https://doi.org/10.1007/978-3-319-18857-7_3

Crespi-Vallbona, M., y Mascarilla-Miró, O. (2020). Wine lovers: their interests in tourist experiences. International Journal of Culture, Tourism, and Hospitality Research, 14(2), 239-258.

Dallen, J. T. (2005). Shopping tourism, retailing and leisure. In Shopping Tourism, Retailing and Leisure. https://doi.org/10.1108/ IJCTHR-05-2019-0095

Davidson, R., y Keup, M. (2014). The Use of Web 2.0 as a Marketing Tool by European Convention Bureaux. Scandinavian Journal of Hospitality and Tourism. https://doi.org/10.1080/15022250.2014.946232

Davies, M., Musango, J. K., \& Brent, A. C. (2016). A systems approach to understanding the effect of Facebook use on the quality of interpersonal communication. Technology in Society. https://doi.org/10.1016/j.techsoc.2015.10.003

Dellarocas, C. (2003). The digitization of word of mouth: Promise and challenges of online feedback mechanisms. Management Science. https://doi.org/10.1287/mnsc.49.10.1407.17308

Delplanque, S., Grandjean, D., Chrea, C., Aymard, L., Cayeux, I., Le Calve, B., \& Sander, D.(2008). Emotional processing of odors: Evidence for a nonlinear relation betweenpleasantness and familiarity evaluations.Chemical Senses, 33(5), 469-479

Dimanche, F. (2003). The Louisiana tax free shopping program for international visitors: a case study. Journal of Travel Research, 41(3), 311-314. https://doi.org/10.1177/0047287502239044

Dodd, T. H. (1999). Attracting Repeat Customers to Wineries. International Journal of Wine Marketing. https://doi.org/10.1108/ eb008693

Dolan, R., y Goodman, S. (2017). Succeeding on social media: Exploring communication strategies for wine marketing. Journal of Hospitality and Tourism Management, 33, 23-30. https://doi.org/10.1016/j.jhtm.2017.09.001

Dougherty, P. H. (2012). The geography of wine: Regions, terroir and techniques. In The Geography of Wine: Regions, Terroir and Techniques. https://doi.org/10.1007/978-94-007-0464-0

Elias Pastor, L. V. (2006). El turismo del vino. Otra experiencia de ocio. In Documentos de estudios de ocio. Retrieved from http:// www.deusto-publicaciones.es/deusto/pdfs/ocio/ocio30.pdf

Espejel, J., Fandos, C., y Flavián, C. 2011). Antecedents of Consumer Commitment to a PDO Wine: An Empirical Analysis of Spanish Consumers. Journal of Wine Research. https://doi.org/10.1080/09571264.2011.622516 
Fatanti, M. N., y Suyadnya, I. W. (2015). Beyond user gaze: How Instagram creates tourism destination brand?. Procedia-Social and Behavioral Sciences, 211, 1089-1095.

Fernandes, T., y Cruz, M. (2016). Dimensions and outcomes of experience quality in tourism: The case of Port wine cellars. Journal of Retailing and Consumer Services, 31, 371-379. https://doi.org/10.1016/j.jretconser.2016.05.002

Fernández-Cavia, J., Vinyals-Mirabent, S., Fernández-Planells, A., Weber, W., y Pedraza-Jiménez, R. (2020). Fuentes de información para los turistas en las distintas etapas del viaje. Profesional De La Información, 29(2). https://doi.org/10.3145/epi.2020.mar.19

Festa, G., Shams, S. M. R., Metallo, G., \& Cuomo, M. T. (2020). Opportunities and challenges in the contribution of wine routes to wine tourism in Italy - A stakeholders' perspective of development. Tourism Management Perspectives. https://doi.org/10.1016/j. tmp.2019.100585

Flavián, C., Ibáñez-Sánchez, S., y Orús, C. (2018). The impact of virtual, augmented and mixed reality technologies on the customer experience. Journal of Business Research, 100(January 2018), 547-560. https://doi.org/10.1016/j.jbusres.2018.10.050

Fondevila-Gascón, J. F., Gutiérrez-Aragón, Ó., Copeiro, M., Villalba-Palacín, V., \& Polo-López, M. (2020). Influencia de las historias de Instagram en la atención y emoción según el género. Comunicar, 28(63), 41-50.

Fuentes, R. C., Moreno-Gil, S., González, C. L., y Ritchie, J. B. (2015). La creación y promoción de experiencias en un destino turístico. Un análisis de la investigación y necesidades de actuación. Cuadernos de turismo, (35), 71-94.

Gallace, A., Ngo, M. K., Sulaitis, J., y Spence, C. (2011). Multisensory presence in virtual reality: Possibilities y limitations. In Multiple Sensorial Media Advances and Applications: New Developments in MulSeMedia.

Getz, D. (2000). Explore wine tourism: management, development, destinations. New York: Cognizant

Getz, D., Brown, G. (2006). Critical success factors for wine tourism regions: A demand analysis. Tourism Management. https:// doi.org/10.1016/j.tourman.2004.08.002

Getz, D., Brown, G. (2006b). Benchmarking wine tourism development: The case of the Okanagan Valley, British Columbia, Canada. International Journal of Wine Marketing. https://doi.org/10.1108/09547540610681077

Geurin-Eagleman, A. N., \& Burch, L. M. (2016). Communicating via photographs: A gendered analysis of Olympic athletes' visual self-presentation on Instagram. Sport Management Review. https://doi.org/10.1016/j.smr.2015.03.002

Gil-Rubio, Á., Jimenez-Barandalla, I. C., \& Mercado-Idoeta, C. (2017). Reputación corporativa online en la hotelería: el caso TripAdvisor. Esic Market Economic and Business Journal, 48(3), 595-608. https://www.academia.edu/download/57397510/ ReputacionESIC.pdf

Godes, D.,y Mayzlin, D. (2004). Using online conversations to study word-of-mouth communication. Marketing science, 23(4), 545-560. https://doi.org/10.1287/mksc.1040.0071

Gold, D., Katz, E., Lazarsfeld, P. F., y Roper, E. (1956). Personal Influence: The Part Played by People in the Flow of Mass Communications. American Sociological Review. 
Gonçalves, R., Martins, J., Pereira, J., Cota, M., y Branco, F. (2016). Promoting e-commerce software platforms adoption as a means to overcome domestic crises: The cases of portugal and spain approached from a focus-group perspective. Advances in Intelligent Systems and Computing.

Goyette, I., Ricard, L., Bergeron, J., y Marticotte, F. (2010). E-WOM scale: Word-of-mouth measurement scale for e-services context. Canadian Journal of Administrative Sciences.

Gretzel, U., Zhong, L., y Koo, C. (2016). Application of smart tourism to cities. International Journal of Tourism Cities. Springer, Cham. https://doi.org/10.1007/978-3-319-26285-7_22

Hall, C. M. (2013). Wine, food, and tourism marketing. Routledge.

Hall, C.M.C.; Macionis, N. (1998). Wine tourism in Australia and New Zealand. In Tourism and Recreation in Rural Areas; Butler., R.W., Hall, C.M., Jenkins, J.M., Eds.; Wiley and Sons: Chichester, UK, 197-224.

Hall, C. M., Johnson, G., Cambourne, B., Macionis, N., Mitchell, R., y Sharples, L. (2009). Wine tourism: an introduction. In Wine tourism around the world (pp. 1-23), Butterworth-Heinemann, Oxford. https://doi.org/10.4324/9780080521145-13.

Hall, C. M., Longo, A. M., Mitchell, R., y Johnson, G. (1996, December). Wine tourism in new Zealand. In Proceedings of tourism down under II: A research conference (pp. 109-119). Dunedin: University of Otago.

Hall, C. M., y Mitchell, R. (2002). The tourist terroir of New Zealand wine: The importance of region in the wine tourism experience. Societa Geografica Italiana. Roma.

Hall, C. M., y Mitchell, R. (2007). Wine marketing: A practical guide. In Wine Marketing: A Practical Guide. ButterworthHeinemann, Oxford.

Hall, C.M., Sharples, L., Cambourne y B., Macionis, (2000). Wine tourism around the world. Butterworth-Heinemann, Oxford.

Hall, C. M., Sharples, L., Cambourne, B., Macionis, N., Telfer, D. J. (2019). The Northeast Wine Route: wine tourism in Ontario, Canada and New York State. In Wine Tourism Around the World. https://doi.org/10.4324/9780080521145-13

Hart, C, (1998). Doing a Literature Review: Releasing the Social Science Research Imagination; Sage: New York.

Hennig-Thurau, T., Gwinner, K. P., Walsh, G., y Gremler, D. D. (2004). Electronic word-of-mouth via consumer-opinion platforms: What motivates consumers to articulate themselves on the Internet? Journal of Interactive Marketing. https://doi.org/10.1002/ dir. 10073

Heung, V. C. S., y Cheng, E. (2000). Assessing tourists' satisfaction with shopping in the Hong Kong special administrative region of China. Journal of Travel Research. https://doi.org/10.1177/004728750003800408.

Hojman, D. E., Hunter-Jones, P. (2012). Wine tourism: Chilean wine regions and routes. Journal of Business Research. https://doi. org/10.1016/j.jbusres.2011.07.009

Holbrook, M. B., y Hirschman, E. C. (1982). The Experiential Aspects of Consumption: Consumer Fantasies, Feelings, and Fun. Journal of Consumer Research.

Hosany, S., y Witham, M. (2010). Dimensions of cruisers' experiences, satisfaction, and intention to recommend. Journal of Travel Research. https://doi.org/978-1-905846-29-0. 
Howell, R. T., y Guevarra, D. A. (2013). Buyinh Happiness: Differential Consumption Experiences for Material and Experiential Purchases (A. M. Columbus (Ed.); Issue July). Advances in Pshycology Research.

Huang, Y. C., Backman, S. J., Backman, K. F., y Moore, D. W. (2013). Exploring user acceptance of 3D virtual worlds in travel and tourism marketing. Tourism Management. https://doi.org/10.1016/j.tourman.2012.09.009.

Huang, Y. C., Backman, K. F., Backman, S. J., y Chang, L. L. (2016). Exploring the Implications of Virtual Reality Technology in Tourism Marketing: An Integrated Research Framework. International Journal of Tourism Research. https://doi.org/10.1002/ jtr.2038

Jalilvand, M. R., Esfahani, S. S., y Samiei, N. (2011). Electronic word-of-mouth: Challenges and opportunities. Procedia Computer Science, 3, 42-46. https://doi.org/10.1016/j.procs.2010.12.008

Jung, D., \& Cho, M.-H. (2015). A Discovery of the Positive Travel Experience in Pre-Trip, On-Site and Post-Trip Stage. Tourism Travel and Research Association: Advancing Tourism Research Globally., 1-6.

Karimi, S. (2013). A purchase decision-making process model of online consumers and its influential factor a cross sector analysis. Tesis Doctoral. In University of Manchester. https://www.escholar.manchester.ac.uk/api/datastream?publicationPid=uk-acman-scw:189583\&datastreamId=FULL-TEXT.PDF

Kempf, D. A. S. (1999). Attitude formation from product trial: Distinct roles of cognition and affect for hedonic and functional products. Psychology and Marketing. https://doi.org/10.1002/(SICI)1520-6793(199901)16:1<35::AID-MAR3>3.0.CO;2-U

Khartishvili, L., Muhar, A., Dax, T., \& Khelashvili, I. (2019). Rural tourism in Georgia in transition: Challenges for regional sustainability. Sustainability (Switzerland). https://doi.org/10.3390/su11020410

Kim, S., y Littrell, M. A. (2001). Souvenir buying intentions for self versus others. Annals of Tourism Research. https://doi. org/10.1016/S0160-7383(00)00064-5

Kirkman, A., Strydom, J.W. \& Van Zyl, C. (2013). Stellenbosch Wine Route wineriers: Management's perspective on the advantages and key success factors of wine tourism. Southern African Business Review.

Koch, J., Martin, A., \& Nash, R. (2013). Overview of perceptions of German wine tourism from the winery perspective. International Journal of Wine Business Research. https://doi.org/10.1108/17511061311317309

Költringer, C., y Dickinger, A. (2015). Analyzing destination branding and image from online sources: A web content mining approach. Journal of Business Research. https://doi.org/10.1016/j.jbusres.2015.01.011

Kotler, P., Bowen, J. T., Makens, J.y Baloglu, S. (2017). Marketing for Hospitality and Tourism. Boston, MA: Pearson Education.

Ku, E. C. S., y Chen, C. Der., (2015) Cultivating travellers' revisit intention to e-tourism service: The moderating effect of website interactivity. Behaviour and Information Technology, 34(5), 465-478. https://doi.org/10.1080/0144929X.2014.978376

Ladhari, R., y Michaud, M. (2015). EWOM effects on hotel booking intentions, attitudes, trust, and website perceptions. International Journal of Hospitality Management. https://doi.org/10.1016/j.ijhm.2015.01.010

Laverie, D. A., Humphrey, W. F., Velikova, N., Dodd, T. H., y Wilcox, J. B. (2011). Building wine brand communities with the use of social media: A conceptual model. In Proceedings of the 6th AWBR International Conference (pp. 9-10). 
Law, R., Buhalis, D., y Cobanoglu, C. (2014). Progress on information and communication technologies in hospitality and tourism. International Journal of Contemporary Hospitality Management. https://doi.org/10.1108/IJCHM-08-2013-0367

Lewis, D. y Bridger D., (2001) The soul of the new consumer: Authenticity - What we buy and why in the new economy. Nicholas Brealey Publishing. https://doi.org/10.1108/jcm.2001.18.2.179.2

Lin, L., y Mao, P.-C. (2015) Food for memories and culture - A content analysis study of food specialties and souvenirs. Journal of Hospitality and Tourism Management, 22, 19-29. https://doi.org/10.1016/j.jhtm.2014.12.001

Litvin, S. W., Goldsmith, R. E., y Pan, B. (2008). Electronic word-of-mouth in hospitality and tourism management. Tourism Management. https://doi.org/10.1016/j.tourman.2007.05.011

Llodrà-Riera, I., Martínez-Ruiz, M. P., Jiménez-Zarco, A. I., y Izquierdo-Yusta, A. (2015). A multidimensional analysis of the information sources construct and its relevance for destination image formation. Tourism Management. https://doi.org/10.1016/j. tourman.2014.11.012

López-Guzmán, T., Garcia, J. R., \& Rodriguez, Á. V. (2013). Revisión de la literatura cientifica sobre enoturismo en españa. Cuadernos de Turismo.

Lourenço-Gomes, L., Pinto, L. M. C., \& Rebelo, J. (2015). Wine and cultural heritage. the experience of the Alto Douro Wine Region. Wine Economics and Policy. https://doi.org/10.1016/j.wep.2015.09.001

Lu, W., y Stepchenkova, S. (2012). Ecotourism experiences reported online: Classification of satisfaction attributes. Tourism Management. https://doi.org/10.1016/j.tourman.2011.08.003

Mak, A. H. N. (2017). Online destination image: Comparing national tourism organisation's and tourists' perspectives. Tourism Management. https://doi.org/10.1016/j.tourman.2016.12.012

Mănilă, M. (2012). Wine tourism-a great tourism offer face to new challenges. Revista de turism-studii si cercetari in turism, (13). http://revistadeturism.ro/rdt/article/view/13/9

Marasco, A., Buonincontri, P., van Niekerk, M., Orlowski, M., y Okumus, F. (2018). Exploring the role of next-generation virtual technologies in destination marketing. Journal of Destination Marketing and Management. https://doi.org/10.1016/j. jdmm.2017.12.002

Mariani, M. M., Di Felice, M., y Mura, M. (2016). Facebook as a destination marketing tool: Evidence from Italian regional Destination Management Organizations. Tourism Management. https://doi.org/10.1016/j.tourman.2015.12.008.

Mário, C., \& Tânia, R. (2011). Emarketing for Wine Tourism. Enlightening Tourism. A Pathmaking Journal, 1(1), 93-110. http:// hdl.handle.net/10400.26/4349.

Martins, J., Gonçalves, R., Branco, F., Barbosa, L., Melo, M., y Bessa, M. (2017). A multisensory virtual experience model for thematic tourism: A Port wine tourism application proposal. Journal of Destination Marketing and Management. https://doi. org/10.1016/j.jdmm.2017.02.002.

McLuhan, R. (2000). Go live with a big brand experience. Marketing, 26(4), 45-46. 
Meng, F., y Xu, Y. (2012). Tourism shopping behavior: Planned, impulsive, or experiential? International Journal of Culture, Tourism and Hospitality Research, 6(3), 250-265. https://doi.org/10.1108/17506181211246401.

Millán Vázquez De La Torre, G., Morales Fernández, E., y Pérez Naranjo, L. M. (2014). Turismo gastronómico, Denominaciones de Origen y desarrollo rural en Andalucía: situación actual. Boletín de La Asociación de Geógrafos Españoles, 65, 113-138.

Mitchell, T. R., Thompson, L., Peterson, E., y Cronk, R. (1997). Temporal Adjustments in the Evaluation of Events: The "Rosy View." Journal of Experimental Social Psychology. https://doi.org/10.1006/jesp.1997.1333

Mitchell, R., y Hall, C. M. (2004). The post-visit consumer of New Zealand winery visitors. Journal of Wine Research, 15(1), 39-49. https://doi.org/10.1080/0957126042000300317.

Mitchell, R., Hall, C. M. (2006). Wine tourism research: the state of play. Tourism review international, 9(4), 307-332. https://doi. org/10.3727/154427206776330535.

Molinillo, S., Liébana-Cabanillas, F., Anaya-Sánchez, R., y Buhalis, D. (2018). DMO online platforms: Image and intention to visit. Tourism Management, 65, 116-130. https://doi.org/10.1016/j.tourman.2017.09.021.

Montella, M. M. (2017). “Wine Tourism and Sustainability: A Review” Sustainability 9, 1: 113. https://doi.org/10.3390/su9010113 Mulrow, C.D. (1994). Rationale for systematic reviews. Br. Med. J. 309, 597-599. https://doi.org/10.1136/bmj.309.6954.597

Nawijn, J., Marchand, M. A., Veenhoven, R., y Vingerhoets, A. J. (2010). Vacationers happier, but most not happier after a holiday. Applied Research in Quality of Life. 5, 35-47 (2010). https://doi.org/10.1007/s11482-009-9091-9.

O’Neill, M., Palmer, A., y Charters, S. (2002). Wine production as a service experience - the effects of service quality on wine sales. Journal of Services Marketing. https://doi.org/10.1108/08876040210433239.

O'Neill, M., Charters, S. (2006). Service quality at the cellar door: A lesson in services marketing from Western Australia's winetourism sector. In Managing Tourism and Hospitality Services: Theory and International Applications. CABI, Wallingford, 251-61.

Pan, F. C., su, S. J., y Chiang, C. C. (2008). Dual attractiveness of winery: Atmospheric cues on purchasing. International Journal of Wine Business Research. https://doi.org/10.1108/17511060810883731.

Perry Hobson, J. S., y Williams, A. P. (1995). Virtual reality: A new horizon for the tourism industry. Journal of Vacation Marketing. https://doi.org/10.1177/135676679500100202.

Piron, F. (1993). A comparison of emotional reactions experienced by planned, unplanned and impulse purchasers. Advances in Consumer Research.

Privitera, D. (2010). Heritage and wine as tourist attractions in rural areas. 116th EAAE Seminar" Spatial Dynamics In Agriculture. Relevent, Case Studies: Teleporter. (2014). https://www.relevent.com/work/marriott/teleporter (Último acceso 06/05/2020)

Rodríguez-Molina, M. A., Frías-Jamilena, D. M., y Castañeda-García, J. A. (2015). The contribution of website design to the generation of tourist destination image: The moderating effect of involvement. Tourism Management. https://doi.org/10.1016/j. tourman.2014.10.001. 
Rodríguez Zulaica, A. (2016). ¿ Cómo diseñar una experiencia turística?. ¿ Cómo diseñar una experiencia turística?, Editorial UOC, Barcelona.

Ruiz-Romero de la Cruz, E., Zamarreño-Aramendia, G., The Sustainability of the Territory and Tourism Diversification: A Comparative Analysis of the Profile of the Traditional and the Oenologic Tourist Through the Future Route of Wine in Malaga. Journal of Business and Economics. 11(1), 22-41. https://doi.org/10.15341/jbe(2155-7950)/01.11.2020/003

Rook, D. W., y Fisher, R. J. (1995). Normative Influences on Impulsive Buying Behavior. Journal of Consumer Research. https:// doi.org/10.1086/209452

Santos, V. R., Ramos, P., Almeida, N., y Santos-Pavón, E. (2019). Wine and wine tourism experience: a theoretical and conceptual review. Worldwide Hospitality and Tourism Themes, 11(6), 718-730. https://doi.org/10.1108/WHATT-09-2019-0053.

Sellers-Rubio, R., Nicolau-Gonzalbez, J. L. (2016). Estimating the willingness to pay for a sustainable wine using a Heckit model. Wine Economics and Policy. https://doi.org/10.1016/j.wep.2016.09.002.

Senecal, S., y Nantel, J. (2004). The influence of online product recommendations on consumers' online choices. Journal of Retailing. 80 (2), 159-169. https://doi.org/10.1016/j.jretai.2004.04.001

Schlüter, R. G., \& Norrild, J. (2015). Enotourism in Argentina: The power of wine to promote a region. In Tourism in Latin America: Cases of Success. https://doi.org/10.1007/978-3-319-05735-4_4

Shen, S., Sotiriadis, M., Zhang, Y. (2020). The influence of smart technologies on visit experience / customer journey in tourist attractions within the smart tourism management framework, Sustainability 12 (10). https://doi.org/10.3390/su12104157.

Shu, L. (2014). For Marriott, the future of travel is a virtual-reality teleporter phone booth. Digital Trends, Retrieved on, 7(09) https://www.digitaltrends.com/photography/marriott-oculus-rift-virtual-real-world-application (último acceso 08/05/2020)

Sigala, M. (2014). Wine tourism around the world: development, management and markets. Journal of Wine Research.https://doi. org/10.1080/09571264.2014.907136

Stepchenkova, S., y Li, X. (2014). Destination image: Do top-of-mind associations say it all? Annals of Tourism Research. 45. 4262- https://doi.org/10.1016/j.annals.2013.12.004.

Stepchenkova, S., y Zhan, F. (2013). Visual destination images of Peru: Comparative content analysis of DMO and user-generated photography. Tourism Management. 36, 590-601. https://doi.org/10.1016/j.tourman.2012.08.006

Swanson, K. K. (2004). Tourists' and retailers' perceptions of souvenirs. Journal of Vacation Marketing. https://doi. org/10.1177/135676670401000407.

Tranfield, D., Denyer, D., Smart, P. (2003). Towards a Methodology for Developing Evidence-Informed Management Knowledge by Means of Systematic Review. In British Journal of Management. https://doi.org/10.1111/1467-8551.00375.

Trišić, I., Štetić, S., Privitera, D., Nedelcu, A. (2020). Wine routes in Vojvodina Province, Northern Serbia: A tool for sustainable tourism development. Sustainability (Switzerland). https://doi.org/10.3390/SU1201008

Tussyadiah, I. P., Wang, D., Jung, T. H., y tom Dieck, M. C. (2018). Virtual reality, presence, and attitude change: Empirical evidence from tourism. Tourism Management. https://doi.org/10.1016/j.tourman.2017.12.003 
Williams, P. W., Kelly, J. (2001). Cultural Wine Tourists: Product Development Considerations for British Columbia's Resident Wine Tourism Market. International Journal of Wine Marketing. https://doi.org/10.1108/eb008727.

Wolikow, S. (2014). "Le vin de Champagne: De la visite à l'Oenotourisme, un chemin difficile". CULTUR: Revista de Cultura e Turismo.

$\mathrm{Wu}, \mathrm{M}$. H. (2013). Relationships among source credibility of electronic word of mouth, perceived risk, and consumer behavior on consumer generated media. UMassAmherst. https://scholarworks.umass.edu/cgi/viewcontent.cgi? article=2123\&context=theses Xiong, J., Hashim, N. H., y Murphy, J. (2015). Multisensory image as a component of destination image. Tourism Management Perspectives, 14, 34-41. https://doi.org/10.1016/j.tmp.2015.03.001

Yang, C., Huang, C. R., y Yang, K. C. (2011). eWOM: The effects of online consumer information adoption on purchasing decision. In Marketing Trends Kongress.

Yim, M. Y. C., Chu, S. C. and Sauer, P. L. (2017). Is Augmented Reality Technology an Effective Tool for E-commerce? An Interactivity and Vividness Perspective. Journal of Interactive Marketing, 39, 89-103. https://doi.org/10.1016/j.intmar.2017.04.001

Yuan, J. J., Morrison, A. M., Linton, S., Feng, R., \& Jeon, S. M. (2004). Marketing small wineries: An exploratory approach to website evaluation. Tourism Recreation Research. https://doi.org/10.1080/02508281.2004.11081453.

Yu, H., y Littrell, M. A. (2005). Tourists' Shopping Orientations for Handcrafts. Journal of Travel y Tourism Marketing. https://doi. org/10.1300/J073v18n04_01.

Zamarreño-Aramendia, Gorka; Cruz-Ruiz, Elena; Ruiz-Romero de la Cruz, Elena. 2021. Sustainable Economy and Development of the Rural Territory: Proposal of Wine Tourism Itineraries in La Axarquía of Malaga (Spain). Economies 9, 1:29. https://doi. org/10.3390/economies9010029

Zeng, B., y Gerritsen, R. (2014). What do we know about social media in tourism? A review. In Tourism Man 
